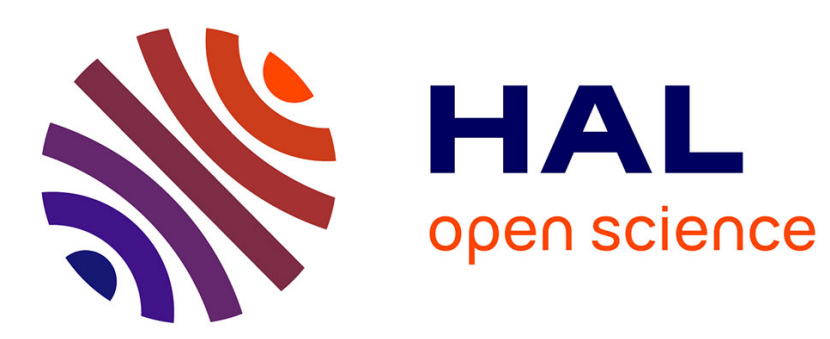

\title{
Electric field gradients in continuous random networks simulating amorphous fluoride FeF3
}

\author{
Jean-Marc Greneche, F. Varret, J. Teillet
}

\section{To cite this version:}

Jean-Marc Greneche, F. Varret, J. Teillet. Electric field gradients in continuous random networks simulating amorphous fluoride FeF3. Journal de Physique, 1988, 49 (2), pp.243-248. 10.1051/jphys:01988004902024300 . jpa-00210690

\section{HAL Id: jpa-00210690 https://hal.science/jpa-00210690}

Submitted on 1 Jan 1988

HAL is a multi-disciplinary open access archive for the deposit and dissemination of scientific research documents, whether they are published or not. The documents may come from teaching and research institutions in France or abroad, or from public or private research centers.
L'archive ouverte pluridisciplinaire HAL, est destinée au dépôt et à la diffusion de documents scientifiques de niveau recherche, publiés ou non, émanant des établissements d'enseignement et de recherche français ou étrangers, des laboratoires publics ou privés. 
Classification

Physics Abstracts

$76.80-61.40-61.90$

\title{
Electric field gradients in continuous random networks simulating amorphous fluoride $\mathrm{FeF}_{3}$
}

\author{
J. M. Greneche $\left({ }^{1}\right)$, F. Varret $\left({ }^{1}\right)$ and J. Teillet $\left(^{2}\right)$ \\ (1) Laboratoire de Spectrométrie Mössbauer, UA 807, Université du Maine, 72017 Le Mans, France \\ (') L.P.C.M., Institut National des Sciences Appliquées, 76130 Mont-Saint-Aignan, France
}

(Reçu le 12 février 1987, accepté sous forme définitive le 14 octobre 1987)

\begin{abstract}
Résumé. - Les calculs de gradients de champ électrique ont été développés pour des modèles de structure amorphe en utilisant un modèle de charges ponctuelles par intégration sur des couronnes sphériques. Les structures considérées résultent de modèles continus aléatoires d'octaèdres joints par les sommets simulant un amorphe type $\mathrm{FeF}_{3}$. La distribution de séparation quadripolaire déduite de ces calculs est en bon accord avec les données expérimentales obtenues par spectrométrie Mössbauer avec des composantes équiprobables de gradients positifs et négatifs. Ces résultats sont discutés en termes d'arrangements locaux et de distorsions d'octaèdres.
\end{abstract}

\begin{abstract}
Electric field gradient calculations have been developed in amorphous structures using a point charge model. The summations were performed on successive spherical shells. The structures under study were continuous random networks of corner-sharing octahedra simulating amorphous $\mathrm{FeF}_{3}$. The computed quadrupole splitting distribution compares well to experimental data from Mössbauer Spectroscopy. Both positive and negative gradients are found, with equal probability, also in agreement with Mössbauer data on $\mathrm{FeF}_{3}$. These results are discussed in terms of local ionic arrangements and distorted octahedra.
\end{abstract}

\section{Introduction.}

The structural properties of amorphous materials have been extensively studied since some years. Chemical and positional order at short and medium ranges are investigated by scattering experiments such as neutrons, X-rays, E.X.A.F.S., etc. Unfortunately these techniques are restricted to radial information because of the macroscopic isotropy of the samples. Therefore, other techniques are needed to approach many-atom correlation functions such as bonding angle distributions for example. So, the electric field gradient (E.F.G.) tensor has revealed to provide useful information concerning local environments of an ion probe, in the range of some interatomic distances.

${ }^{57} \mathrm{Fe}$ Mössbauer spectroscopy is an excellent tool for studying electric quadrupole interactions, through quadrupole splitting $\Delta$ related to E.F.G. components tensor via the following equation (for a nuclear spin 3/2) :

$$
\Delta=\frac{e Q\left|V_{z z}\right|}{2}\left(1+\frac{\eta^{2}}{3}\right)^{1 / 2}
$$

where $V_{z z}$ is the main component of the E.F.G. tensor, $O X Y Z$ the principal axes, $\eta=$ $\left(V_{x x}-V_{y y}\right) / V_{z z}$ the asymmetry parameter and $Q$ the nuclear quadrupole moment; $\eta$ values are restricted to $0 \leqslant \eta \leqslant 1$ with the usual convention : $\left|V_{x x}\right| \leqslant\left|V_{y y}\right| \leqslant\left|V_{z z}\right|$.

The paramagnetic Mössbauer spectra exhibit broad and non-Lorentzian lines because of the local distribution of charges around the probe iron nuclei : the quadrupolar splitting distribution $P(\Delta)$ is derived from such spectra. However, any accurate information concerning the asymmetry parameter $\boldsymbol{\eta}$ cannot be derived by usual means, i.e. high field Mössbauer spectroscopy in the paramagnetic state, because of random induced hyperfine fields [27]. Consequently, the distribution function $P\left(V_{z z}, \eta\right)$ cannot be directly observed from Mössbauer experiments in the present case (this due to the small nuclear spin value $I=3 / 2$ ).

On the other hand, expected distributions $P\left(V_{z z}, \eta\right)$ can be obtained in two ways :

- Czjzek [1] proposed an analytic development established from environment models based on 
random ionic coordinations : the main lines of this approach are given in the following section;

- recently, structural models based on random corner-sharing octahedral networks were developed first by hand and then by computer: here, we present E.F.G. distributions calculated from atomic positions of such a computed model.

These models seem to be better suited to describe amorphous ferric fluorides, which have a well-defined coordination, than to describe the models based on random packing of ionic spheres previously published [2].

\section{Random coordination models.}

Czjzek has shown for solids which are isotropic on the average, that the distribution function of the $V_{i j}$ components of the E.F.G. tensor could be expressed through two invariant functions of the components $V_{i j}\left(S=V_{z z}^{2}\left(1+\eta^{2} / 3\right)\right.$ and $D=$ $\left.V_{z z}^{3}\left(1-\eta^{2}\right)\right)[1]$.

After consideration of random environments made of spherical shells, an analytic approximation for the distribution function $P\left(V_{z z}, \eta\right)$ was established :

$$
\begin{aligned}
& P\left(V_{z z}, \eta\right)=\frac{1}{(2 \pi)^{1 / 2} \sigma^{5}} \times \\
& \times V_{z z}^{4} \eta\left(1-\eta^{2} / 9\right) \exp \left(-\frac{V_{z z}^{2}\left(1+\eta^{2} / 3\right)}{2 \sigma^{2}}\right) .
\end{aligned}
$$

Unfortunately, most experiments did not agree with such a model $[3,4]$. This is due to the structural constraints of the local environment of the probe nuclei in amorphous solids : for instance a given polyhedral coordination. Therefore, in the case of iron probe nuclei, a new form was proposed

$$
P(\Delta)=\frac{\Delta^{d-1}}{\sigma^{d}} \exp -\frac{\Delta^{2}}{2 \sigma^{2}}
$$

where $d$ is the number of independent variables and $d<5$ [5].

From this point of view, isomer shift data in amorphous ferric fluorides show that the $\mathrm{Fe}^{3+}$ ions are surrounded by $\left(F^{-}\right)_{6}$ octahedra, and the distortions of these octahedra can be described by two independent random variables, which leads to the following form

$$
P(\Delta)=\frac{\Delta}{\sigma^{2}} \exp -\frac{\Delta^{2}}{2 \sigma^{2}}
$$

The same expression was also proposed by Coey on the basis of a random Gaussian model of 3 independent distribution functions $P\left(V_{i i}\right)$ coupled by La place's equation as illustrated in figure 1 [6].

This last expression turned out to be in rather good agreement with experimental data obtained on amorphous ferric fluorides $[3,7]$; as well, derived expressions could reasonably fit the data of numerous amorphous solids $[8,9,10,11]$.

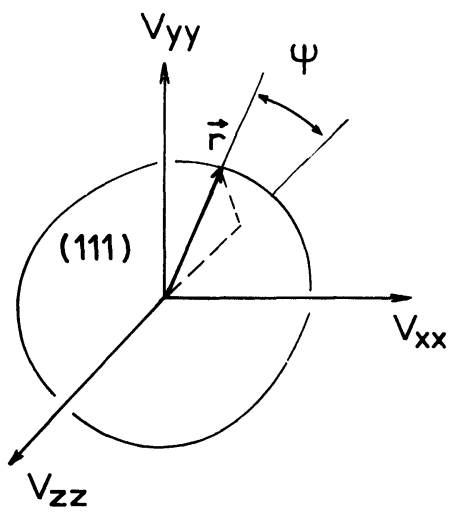

Fig. 1. $-V_{x x}, V_{y y}$ and $V_{z z}$ coordinates : each point of the (111) plane containing the origin corresponds to an E.F.G. tensor.

3. E.F.G. tensor calculations in crystalline structures.

Calculations of electric field gradient for crystalline structures have been extensively carried out for spinels [12, 13], garnets [14, 15, 16] pyrochlores [17] and $\mathrm{AFeF}_{4}$ compounds [18] and agree with experimental data in most cases. The electric field gradient is often calculated using a polarizable point charge model : an ionic crystal is composed of discrete deformable ions located on a three-dimensional periodic lattice. Polarization is due to an electric field acting on the ions considered and this leads to a self-consistent problem.

From a mathematical point of view, the electric field and the E.F.G. tensor are estimated by lattice summations (solving $3 n$ self-consistent equations and $5 n$ equations respectively where $n$ is the number of unequivalent sites in the unit cell).

Some rules have been established by Coogan [19] to obtain absolute convergence in lattice summations : the unit cell has (i) no monopolar moment for the E.F.G. equations; (ii) no monopolar or dipolar moment for the electric field ; (iii) no monopolar, dipolar or quadrupolar moment for the potential.

Furthermore, the calculations require accurate crystallographic data.

In practice, lattice summations have been developed either in direct lattice or in reciprocal lattice. The calculations are carried out on volumes which are multiples of the elementary cell with $(2 L-1)^{3}$ cells symmetrically displayed around the origin cell. Coogan's criteria are easy to satisfy under such conditions for E.F.G. tensor and electric field components ; moreover, an absolute convergence on the $V_{i j}$ component values is rapidly observed as a function of $L$ (for instance after $L=4$ [18]). 
Summations performed on spherical volumes converged more slowly because Coogan's criteria are satisfied only on the average, and are less efficient for computing E.F.G.'s in crystals (for instance sphere radius $\approx 50 \AA$ at least in garnet [16]).

\section{Problems relative to calculations in amorphous structures.}

From a geometrical point of view, the unit cell no longer exists in amorphous solids. The E.F.G. calculations have to be performed on spherical volumes. The dipolar contribution has to be neglected because of the huge number of equations needed; fortunately this contribution is thought to decrease very rapidly as a function of the sphere radius.

Preliminary calculations using spherical shells were performed on a crystalline lattice, including the monopolar contribution only. The limit value was in agreement with calculations using unit cells, but convergence was much slower as it is shown in figure 2; this was clearly associated with large monopolar moments of the spheres. We could greatly speed up convergence - and reduce monopolar moments - by creating a « transitional shell », in which the ion contributions were progressively weighted from the inner to the outer surface of the shell ; the radial dependence of the weighting factor is shown in figure 3.

The influence of the number and thickness of successive shells, and of the thickness of the transi-

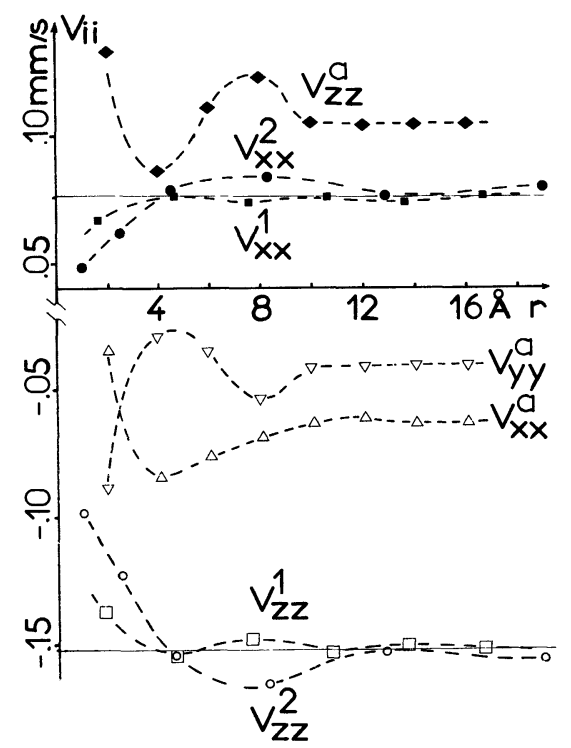

Fig. 2. - Evolution as a function of the integrating sphere radius of the E.F.G. tensor components for crystalline $\mathrm{CsFeF}_{4}$ without considering the transitional shell $\left(V_{i i}\right)^{2}$, with such a shell $\left(V_{i i}^{1}\right)$ and for an iron site of an amorphous network $\left(V_{i i}^{\mathrm{a}}\right)$. Note the expected values calculated in $[17,18]$ for $\mathrm{CsFeF}_{4}: V_{x x}=V_{y y}=0.076 \mathrm{~mm} / \mathrm{s}$ and $V_{z z}=$ $-0.152 \mathrm{~mm} / \mathrm{s}$.

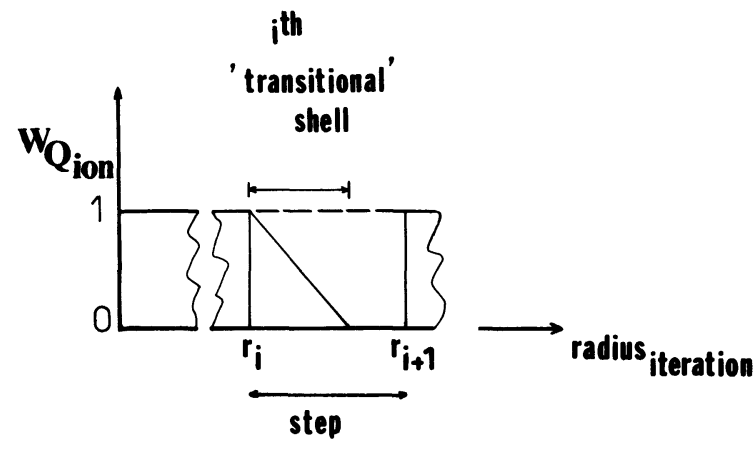

Fig. 3. - Weighting factor of the ion charges reported as a function of the sphere radius.

tional shell were systematically investigated. The optimization of such parameters allowed accurate E.F.G. calculations using a sphere of $10 \AA$ radius at least as shown in figure 4 . Then the deviation of the quadrupole splitting value from the expected limit value was smaller than the typical step of experimental quadrupolar splitting distribution [3], $\left(\delta \Delta \sim 0.1 \mathrm{~mm} \mathrm{~s}^{-1}\right)$. Such a large value is needed for analysing the quadrupole splitting distribution; of course, experimental values are much more accurate $\left(\sim 0.01 \mathrm{~mm} \mathrm{~s}^{-1}\right)$ in average, and a similar accuracy is also expected in the present calculations, when averaging random deviations involved for at least 100 different sites in the disordered model (as a matter of fact, 500 sites were typically used in the forthcoming calculations).

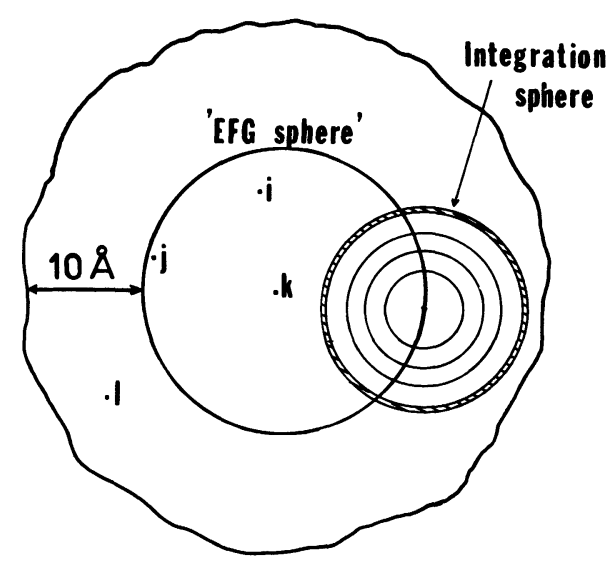

Fig. 4. - Schematic view of the «E.F.G. sphere» containing iron ions on which the E.F.G. numerical integration can be performed.

\section{E.F.G. calculations on random networks.}

5.1 E.F.G. COMPUTATION. - The monopolar contributions of the E.F.G. components are calculated over iron sites inside an «E.F.G. sphere» in the centre of the whole volume of the simulated model (see Fig. 4), to account for the integration volume 
needed around each iron site. Then the E.F.G. tensors are diagonalized by a Jacobi method to obtain the main components $V_{x x}, V_{y y}$ and $V_{z z}$, with $\left|V_{x x}\right| \leqslant\left|V_{y y}\right| \leqslant\left|V_{z z}\right|$, and the set of principal axes. The relevant Mössbauer parameters are deduced for all the sites considered : the asymmetry parameter $\eta$, the quadrupolar splitting value $\Delta$ and the polar angles $(\vartheta, \varphi)$ of the principal E.F.G. axes $\mathrm{O} Z$ with respect to the network reference. The set of data is analysed, by histogram techniques, in terms of $P(\Delta, \eta), P(\Delta)$ and $P(\eta)$. The quadrupolar splitting distribution is also deduced, as well as that of the asymmetry parameter. In the present calculations, the ${ }^{57} \mathrm{Fe}$ quadrupole moment $Q$ is assumed to be 0.20 barns [20] and the Sternheimer antishielding factor $\left(1-\gamma_{\infty}\right)$ to be 10.14 for $\mathrm{Fe}^{3+}$ [21].

5.2 Results ON a-FeF 3 COEY'S Network. - A continuous random network composed of 516 corner-sharing octahedra was handbuilt by Coey and Murphy a few years ago [22]. E.F.G. calculations were carried out for such a model, using the method described in section 4 with the optimized parameter values. The quadrupolar splitting distribution resulted from an «E.F.G. sphere » of 50 ions only. In spite of this small number, the mean quadrupolar splitting value, the $q$ factor defined as $\left\langle\Delta^{2}\right\rangle /\langle\Delta\rangle^{2}=1.26$ for characterizing the relative width of $P(\Delta)$ [3] and the distribution shape were in good agreement with the experimental data obtained at room temperature $(q=1.30(2)$ in the case of deposited a-FeF $\left.{ }_{3}[23]\right)$.

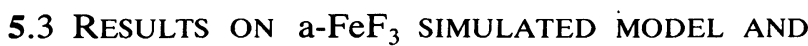
DISCUSSION. - More recently, an algorithm to generate such a continuous random network by computer has been developed and a model composed of $1251 \mathrm{~A}$-atoms $(\mathrm{Fe})$ and $3145 \mathrm{~B}$-atoms $(\mathrm{F})$ was built [24] ; then the «E.F.G. sphere » contained 495 iron sites. Figures 5 and 6 show well resolved distributions $P(\Delta)$ and $P(\eta)$ compared to the theoretical distributions previously predicted by Czjzek; a good agreement with experimental $P(\Delta)$ is noted. The quadrupolar splitting mean value $(\langle\Delta\rangle=0.46 \mathrm{~mm} / \mathrm{s})$ is close to those experimentally obtained on amorphous $\mathrm{FeF}_{3}: 0.55(3) \mathrm{mm} / \mathrm{s}$ for the deposited form and 0.52(2) for the fluorinated variety at room temperature $[23,25]$. One can remark null probability for $\Delta: 0$ or $\eta=0:$ this was predicted for all random coordination models proposed by Czjzek $[1,5]$. Moreover, the marginal $\eta$ distributions (for a given $\Delta$ ) do not sizeably depend on $\Delta$ values but differ from Czjzek's predictions : this difference is well reflected by the most probable $\eta$-value estimated to $\approx 0.6-0.7$ smaller than the value obtained by Czjzek ( 0.8 to 0.9 according the $d$ value).

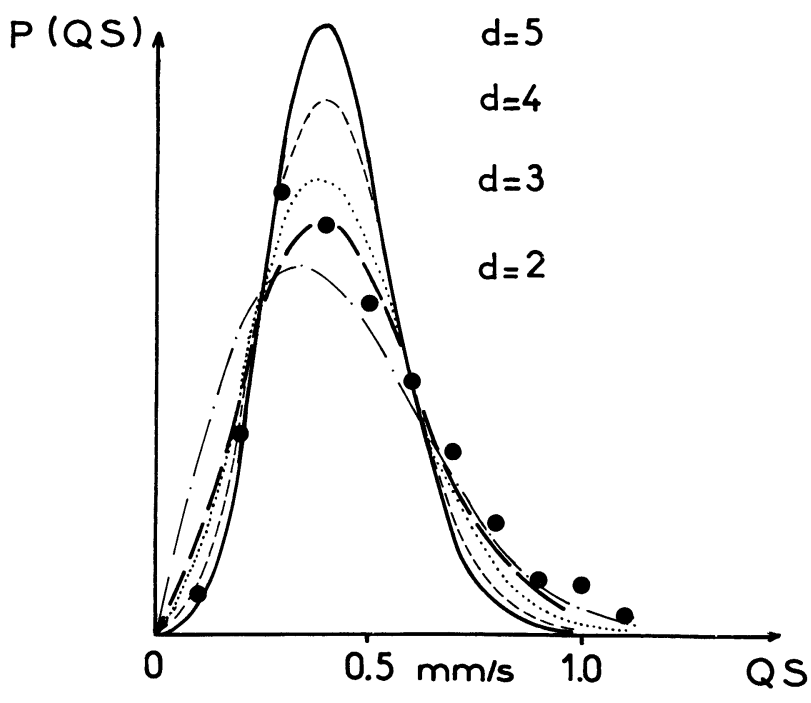

Fig. 5. - Quadrupolar splitting distribution deduced from the simulated random network (squares) ; Czjzek's analytic developments are reported $d=2,3,4$ and 5 and the best fit $d=2.9$ for the present model.

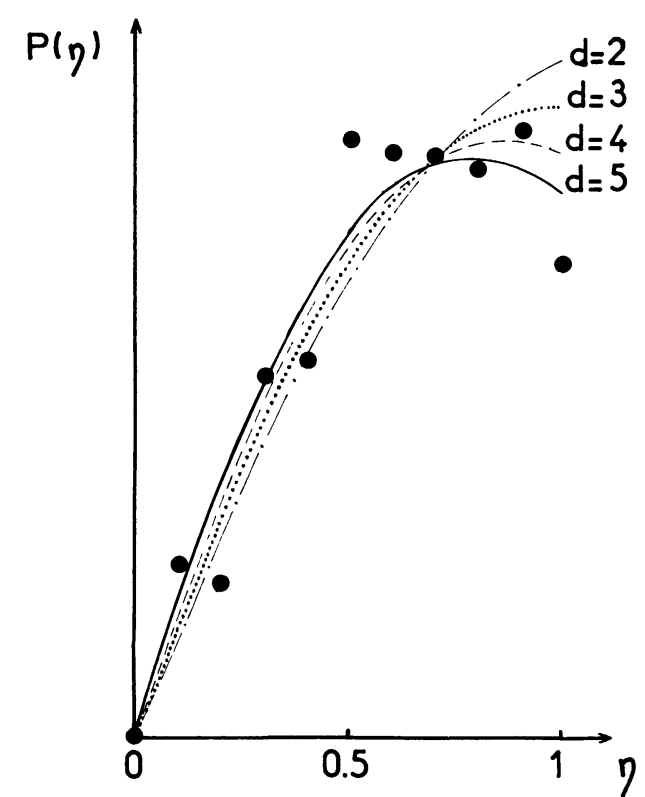

Fig. 6. - Asymmetry parameter distribution calculated on the simulated random network and compared to the previous distributions of Czjzek.

Using the analytic approximation of the distribution function $P(\Delta)$ given by equation (3), the best agreement is obtained with $d=2.9$. However, one can observe in figure 5 that the low $\Delta$ part of the curve could be better reproduced with a larger value of $d$, and the high $\Delta$ part by a smaller one. Such a quadrupolar splitting distribution shape shows a qualitative agreement with the experimental data obtained from Mössbauer spectroscopy at room temperature. The departure could originate either 


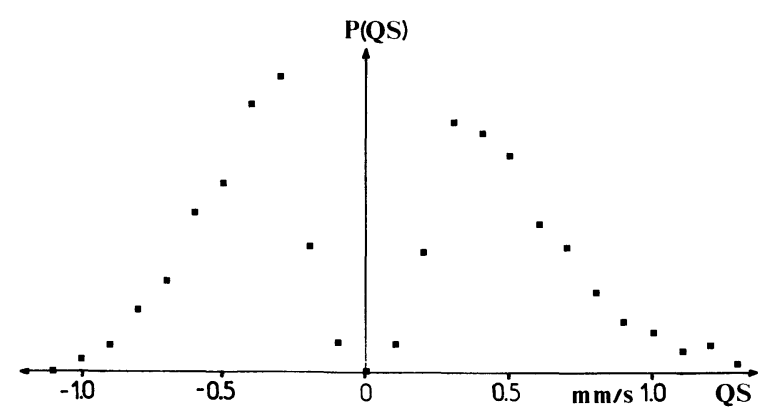

Fig. 7. - Negative and positive contributions to the quadrupolar splitting distribution calculated on the simulated random network.

from the lack of dipolar contributions, or from the limitations of the model. All these remarks suggest that the distortions of the octahedra could be described by two independent variables.

Moreover, the distribution of negative and positive values of $V_{z z}$ are shown in figure 7 in the rate 48 and $52 \%$ respectively: the E.F.G. distribution is not dominantly of a given sign. This result is also supported by experiments : the lineshape analysis of the liquid helium temperature Mössbauer spectrum recorded on a-FeF $\mathrm{F}_{3}$ has revealed nearly equiprobability for finding positive and negative values of $V_{z z}[23,26]$. It is also evidenced by the nearly symmetrical pattern of the spectra recorded under magnetic field above the spin freezing temperature shown in figure 8 ; using a schematic fit which superimposes distributions of axial gradients, positive or negative, and following equation (3) with $d=2$ or 3 , the experimental spectra of deposited $\mathrm{a}-\mathrm{FeF}_{3}$ led to quasi-equal proportions of negative and positive gradients (respectively 55(3) and 45(3) [23]).

Further analysis of these spectra in terms of molecular field distributions will be reported elsewhere [27].

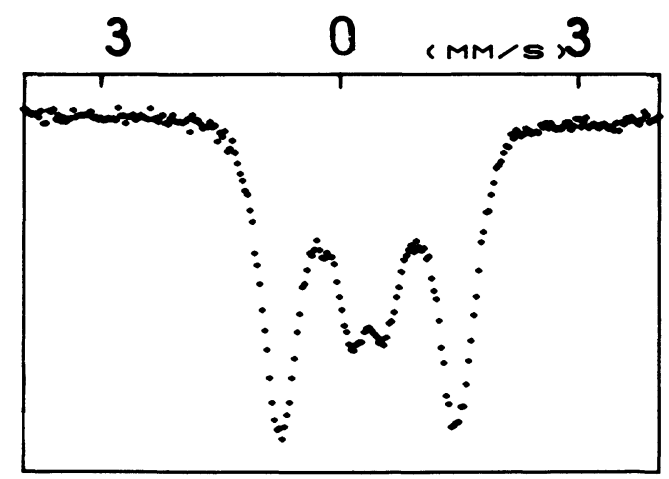

Fig. 8. - Mössbauer spectrum of deposited amorphous $\mathrm{FeF}_{3}$ recorded at $200 \mathrm{~K}$ under $70 \mathrm{kOe}$ applied field parallel to the $\gamma$-beam.
5.4 TOPOlOgy OF THE Distribution $P\left(V_{z z}, \eta\right)$. The topological structure of the $P\left(V_{z z}, \eta\right)$-parameter space can also well be described according to another presentation. The E.F.G. parameters $V_{z z}$ and $\eta$ can be expressed in terms of polar coordinates $(r, \varphi)[28]$ in the following way :

$$
\begin{aligned}
V_{z z} & =-r \cos (\pi / 6+\varphi), \\
\eta & =\sqrt{3} \tan (\pi / 6+\varphi) \text { when } \quad V_{z z}<0
\end{aligned}
$$

and

$$
\begin{aligned}
V_{z z} & =-r \cos (\pi / 6+\varphi), \\
\eta & =\sqrt{3} \tan (\pi / 6+\varphi) \text { when } \quad V_{z z}<0
\end{aligned}
$$

With such a description, all the parameter values $-\infty<V_{z z}<+\infty$ and $0 \leqslant \eta \leqslant 1$ are contained in an adequately selected $60^{\circ}$ sector : this leads to transform the previous $P\left(V_{z z}, \eta\right)$ into an $F(r, \varphi)$ distribution. In the case of the D.R.P.H.S. Czjzek's model, the joint distribution function given by equation (2) takes the following form [28] :

$$
F(r, \varphi)=\frac{r^{4} \cos 3 \varphi}{(2 \pi)^{1 / 2} \sigma^{5}} \exp \left(-r^{2} / 2 \sigma^{2}\right)
$$

Such a function was presented thanks to contour maps in figure 3 in [28] and reproduced in figure 10.1 .

The distribution function deduced from the present model, following the same procedure is shown in figure 9. Such a view confirms a quite good symmetry between positive and negative values of quadrupolar interaction, the presence of a valley centred around $\eta=1$ and the independence between $\eta$ and $V_{z z}$. In addition, figure 10.3 shows the topology of this distribution $P\left(V_{z z}, \eta\right)$ obtained

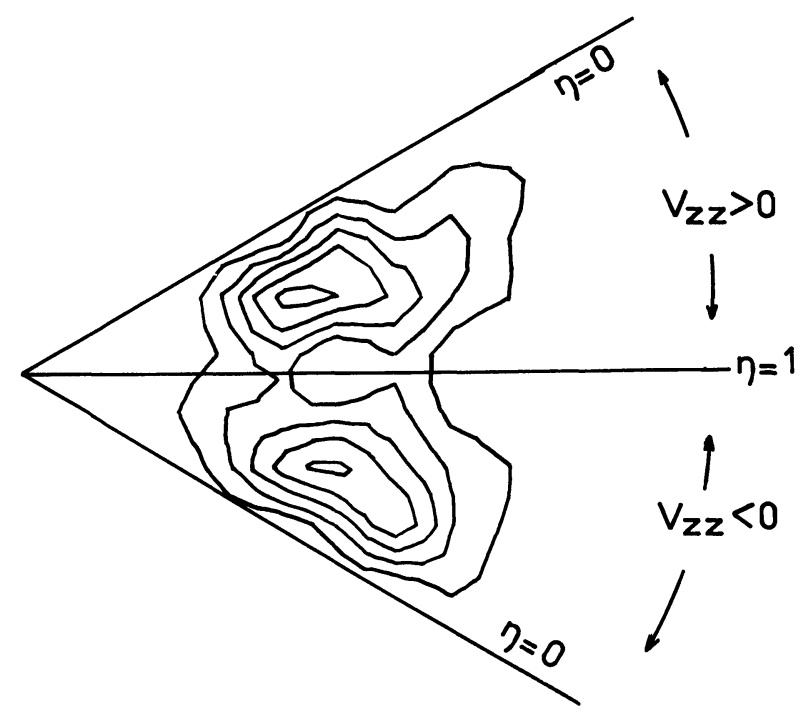

Fig. 9. - Contour maps of $P(r, \varphi)$ figuring the topology of $P\left(V_{z z}, \eta\right)$ calculated on the simulated random network. 


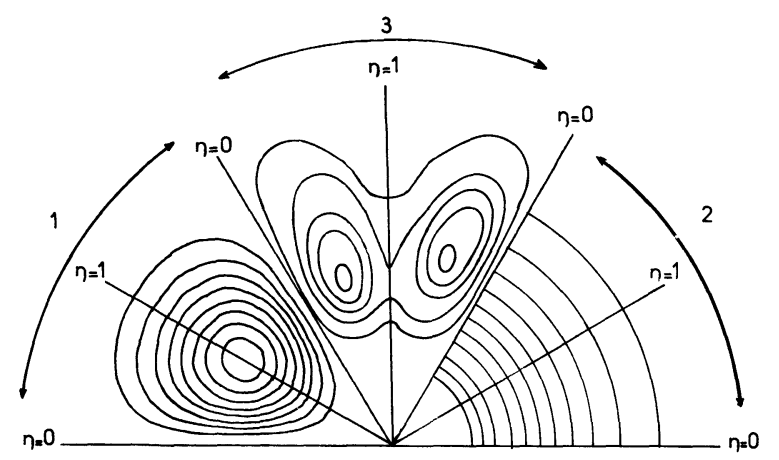

Fig. 10. - Comparison between the $P\left(V_{z z}, \eta\right)$ topological structures of Czjzek's (1), Coey's (2) and the present model, after smoothing (3).

after smoothing of the E.F.G. data : one can remark that the present structural model leads to an intermediate situation between Coey's analysis independent of $\varphi$ and Czjzek's Gaussian developments where no valley appeared around $\eta=1$, for any $d$; the latter point is due to $P(\varphi)$ curves of monotonous shape.

\section{Conclusion.}

The calculations of the components of the E.F.G. tensor using a point charge model were well adapted to the amorphous structures and optimized to increase the «E.F.G. sphere» size. The results obtained from a simulated amorphous network show a qualitative agreement with those deduced from Mössbauer spectra recorded at room temperature on amorphous $\mathrm{FeF}_{3}$ : shape of $P(\Delta),\langle\Delta\rangle$, proportions of negative and positive values of $V_{z z}$. It corroborates the idea that the E.F.G. distribution is basically monitored by the distorted octahedra, through only 2 independent modes. Elsewhere, the $P\left(V_{z z}, \eta\right)$ topology shows that such a model is intermediate between Czjzek's and Coey's predictions. However, the experimental data are not sufficient to give more accurate information concerning the joint distribution $P\left(V_{z z}, \eta\right)$ behaviour.

The random magnetic effects inherent to the amorphous structure prevent us from drawing any more direct structural conclusions from high field Mössbauer spectroscopy experiments in the paramagnetic range but promise indirect conclusions through molecular field distributions.

\section{References}

[1] Czjzek, G., Fink, J., Gotz, F., Schmidt, H., Coey, J. M. D., Rebouillat, J. P. and Lienard, A., Phys. Rev. B 23 (1981) 2513.

[2] Lines, M. E., Phys. Rev. B 21 (1980) 5793.

[3] Lopez-Herrera, M. E., Greneche, J. M. and VArret, F., Phys. Rev. B 28 (1983) 4944.

[4] Maurer, M. and Friedt, J. M., Hyp. Int. 27 (1986) 135.

[5] Czjzek, G., Phys. Rev. B 25 (1982) 4908.

[6] Coey, J. M. D. and CZJZEK, G., unpublished results.

[7] Varret, F. and Henry, M., Revue Phys. Appl. 15 (1980) 1057.

[8] Le Caer, G., Dubois, J. M. and Brand, R. A., Amorphous Metals and Non-equilibrium Processing, MRS Strasbourg (Les Editions de Physique) 1984, p. 249.

[9] Le Caer, G., Cadogan, J. M., Brand, R A., Dubois, J. M. and GunTHERODT, H. J., J. Phys. F 14 (1984) L73.

[10] Friedt, J. M., Maurer, M., Sanchez, J. P. and Durand, J., J. Phys. F 12 (1982) 821.

[11] Maurer, M., Phys. Rev. B 34 (1986) 8996.

[12] Hudson, A. and Whitfield, H. J., Molec. Phys. 12 (1967) 165.

[13] Yagnik, C. M. and Mathur, H. B., Molec. Phys. 16 (1969) 625.
[14] Burns, G., Phys. Rev. 124 (1961) 524.

[15] Nicholson, W. J. and Burns, G., Phys. Rev. A 133 (1964) 1568.

[16] Stadnik, Z. M., J. Phys. Chem. Solids 45 (1984) 311.

[17] Calage, Y. and Pannetier, J., J. Phys. Chem. Solids 38 (1977) 711.

[18] Teillet, J., Calage, Y. and Varret, F., J. Phys. Chem. Solids 43 (1982) 863.

[19] Coogan, C. K., Aust. J. Chem. 20 (1967) 2551.

[20] Stevens, J. G. et al., J. Phys. Chem. 5 (1976) 1093.

[21] Sternheimer, R. M., Phys. Rev. 130 (1963) 1423.

[22] Coey, J. M. D. and Murphy, P. J. K., J. Non Cryst. Solids 50 (1982) 125.

[23] Greneche, J. M., Varret, F., Leblanc, M. and FEREy, G., Solid State Commun. 63 (1987) 435.

[24] Greneche, J. M., Teillet, J. and Coey, J. M. D., J. Physique 47 (1987) 1709.

[25] Lopez-Herrera, M. E., Thesis (1983) Le Mans.

[26] Eibschutz, M., Lines, M. E., VAn Uitert, L. G., GougGenheim, H. J. and ZydZIK, G. J., Phys. Rev. B 29 (1984) 3843.

[27] Greneche, J. M. and Varret, F., Hyp. Int. (accepted).

[28] CzJzeK, G., Hyp. Int. 14 (1984) 189. 